\title{
Analytic NLO Photon Impact Factor for Deep Inelastic Scattering
}

\author{
Giovanni Antonio Chirilli*† \\ CPHT, École Polytechnique, CNRS, 91128 Palaiseau Cedex, France \& \\ LPT, Université Paris-Sud, CNRS, 91405, Orsay, France \\ E-mail: giovanni.chirilliecpht.polytechnique.fr
}

We calculate the photon impact factor for Deep Inelastic Scattering at next-to-leading order using the Operator Product Expansion at high-energy (Regge limit). We expand the T-product of two electromagnetic currents at next-to-leading order in terms of coefficient functions (impact factors) and matrix elements made of Wilson lines operator. The NLO impact factor thus obtained is an analytic expression written as a linear combination of five conformal tensor structures.

35th International Conference of High Energy Physics - ICHEP2010,

July 22-28, 2010

Paris France

\footnotetext{
*Speaker.

${ }^{\dagger}$ The author thanks the organaizers of ICHEP2010 and in particular B. Pire for financial support.
} 
Scattering processes such as Deep Inelastic scattering (DIS) are performed in order to study the structure of the hadronic matter. In the framework of Quantum Chromodynamics (QCD) the T-product of two electromagnetic currents - $\mathrm{T}\left\{j^{\mu}(x) j^{v}(y)\right\}$ - encloses most of the important information of DIS processes. At high-energy (Regge limit) such processes are characterized by the fast increase of the density of the constituents of the target and this behavior is captured in the non-linear Balitsky-Kovchegov evolution equation $[1,2]$ whose linearized version reproduces the BFKL evolution equation. In Regge limit it is natural to introduce as factorization scale the rapidity $Y$ : the amplitude of the process can be represented as a convolution of contributions coming from fields with rapidity $\eta<Y$ ("fast" field) and contributions coming from fields with rapidity $\eta>Y$ ("slow" fields). As in the case of the usual Operator Product expansion (OPE), the integration over fields with rapidity $\eta<Y$ gives us the coefficients function while the integrations over fields with rapidity $\eta>Y$ are the matrix elements of the operators. A general feature of high-energy scattering is that a fast particle moves along its straight-line classical trajectory and the only quantum effect is the eikonal phase factor acquired along this propagation path. In QCD, for a fast quark or gluon scattering off some target, this eikonal factor is a Wilson line: gauge link ordered along the straight line collinear to the particle's velocity $n^{\mu}$. Since particles with very different rapidity perceive each other as Wilson lines such operators are the effective degrees of freedom in high-energy QCD (for a review, see Ref. [4]). The expansion of the T-product of two electromagnetic currents at high-energy (Regge limit) is then written in terms of coefficient functions (impact factors) and matrix element made of Wilson lines operators. In DIS the photon impact factor represents the probability for the virtual photon, exchanged during the scattering process, to split into a quark and anti-quark pair before scattering off the target. Using the high-energy OPE we obtained for the first time an analytic expression of the photon impact factor at NLO accuracy[5], and such result can be expressed as a linear combination of five conformal tensor structures.

\section{References}

[1] I. Balitsky, Nucl. Phys. B463, 99 (1996); "Operator expansion for diffractive high-energy scattering”, [hep-ph/9706411];

[2] Yu.V. Kovchegov, Phys. Rev. D60, 034008 (1999); Phys. Rev. D61,074018 (2000).

[3] V.S. Fadin, E.A. Kuraev, and L.N. Lipatov, Phys. Lett. B 60, (1975) 50; I. Balitsky and L.N. Lipatov, Sov. Journ. Nucl. Phys. 28, (1978) 822.

[4] I. Balitsky, "High-Energy QCD and Wilson Lines", In *Shifman, M. (ed.): At the frontier of particle physics, vol. 2*, p. 1237-1342 (World Scientific, Singapore, 2001) [hep-ph/0101042]

[5] I. Balitsky and G. A. Chirilli, Photon impact factor in the next-to-leading order, arXiv:1009.4729 [hep-ph]. 